\title{
SwissDRG: nécessité d'un monitorage pour une politique de la santé durable
}

Beatrix Meyera,

Heinz Locher ${ }^{b}$, Fred Paccaud ${ }^{c}$

a Service tarifaire $\mathrm{FMH}$, domaine SwissDRG, Olten

b Heinz Locher Management \& Consulting Services, Berne

c IUMSP (Institut universitaire de médecine sociale et préventive) CHUV et Université de Lausanne, Lausanne
Correspondance: Beatrix Meyer Tarifdienst FMH Gösgerstrasse 8 CH-4600 Olten Tel. 0622879696 swissdrg@fmh.ch

\section{Etablir rapidement une recherche concomitante - le temps presse}

$\mathrm{Au} \mathrm{1} 1^{\text {er }}$ janvier $2012 \mathrm{au}$ plus tard, les forfaits par cas seront introduits dans tous les hôpitaux de Suisse. C'est ainsi qu'il en a été décidé lors de la révision de la Loi sur l'assurance-maladie. L'introduction du système de forfaits par cas SwissDRG dans le domaine des soins aigus hospitaliers est associée à beaucoup d'attentes mais aussi à certaines peurs. Les attentes se trouvent en particulier du côté des directeurs de la santé qui espèrent pouvoir identifier, par le biais de SwissDRG, les hôpitaux manquant d'efficacité. Les angoisses sont plutôt émises par les fournisseurs de prestations mais aussi par les organisations de patients, qui craignent une perte de qualité ou que certaines spécialités soient injustement mises sous une pression mettant la sécurité des soins en danger.

Afin de détecter suffisamment tôt les incitatifs erronés, de mettre en place rapidement des mesures adéquates et d'éviter ainsi d'éventuels effets indésirables, la mise sur pied d'une recherche concomitante est indispensable. Cette dernière doit être instaurée un an au plus tard avant l'introduction de SwissDRG, afin de permettre une comparaison «avant-après» de la situation. Et comme le temps presse, la FMH a déjà élaboré, épaulée par des experts, un concept pour la recherche concomitante à l'occasion de l'introduction de SwissDRG [2].

\section{Que faut-il analyser dans le cadre de la recherche concomitante?}

Pour répondre à la question de savoir ce que la recherche concomitante doit exactement analyser, il convient de faire la distinction en trois aspects ou domaines différents: la sécurité des patients et la qualité des soins, la situation des groupes professionnels concernés et le système de santé dans son ensemble. Les tableaux $1 \mathrm{a}$ à $1 \mathrm{c}$ ci-après présentent pour chacun de ces domaines les indicateurs régissant la collecte et l'analyse des données.

\section{SwissDRG SA, I'organisme responsable principal, le déroulement effectif par le Fonds national suisse}

Pour définir la responsabilité dans le cadre de cette recherche, les bases légales disponibles permettent d'envisager différents cas de figure [3]. Ainsi, serait-il pos- sible de confier la responsabilité de ce projet à SwissDRG SA ou à l'Office fédéral de la santé publique (OFSP). Nous recommandons cependant de désigner SwissDRG SA comme organisme principal en charge de la recherche concomitante, tout en laissant la responsabilité générale à l'OFSP pour ce qui concerne l'évaluation des effets de la LAMal. Dans le cadre de la recherche concomitante, le conseil d'administration de SwissDRG SA peut fixer les thèmes à analyser en fonction du concept [4]. Le déroulement de l'activité effective de recherche devrait être confié au Fonds national suisse de la recherche scientifique (FNS). Le FNS se chargerait de la mise au concours. Un jury sélectionné par le FNS, ou un Conseil national de la recherche, pourra vérifier les requêtes soumises et décider laquelle choisir [5]. Cette réglementation permet d'associer les avantages de la pratique et de la légitimation en matière de politique de la santé de SwissDRG SA à la réputation élevée du FNS en sa qualité d'autorité scientifique.

\section{Financement mixte}

Le financement de la recherche concomitante devrait être assuré par différentes sources. Les bases statistiques (en particulier les statistiques médicales et administratives des hôpitaux) et le devoir de contribuer à un registre pourront ainsi être exigés [6]. Les coûts en découlant rentreront dans les coûts d'exploitation des hôpitaux. La garantie des mesures accompagnant la recherche concomitante au sens strict fait partie du mandat aux partenaires tarifaires [7], raison pour laquelle le financement devrait également être assuré par le biais d'une contribution par cas [8]. Le monitorage en général pourra être financé par les moyens que l'OFSP a déjà budgétisés pour l'évaluation des effets de la LAMal [9]. Et finalement, il faudra également faire appel aux sources de financement lié à la recherche mises habituellement à disposition (recherche libre, fonds nationaux, tiers).

\section{Politique de la santé durable grâce à un monitorage à long terme}

Il est important de considérer la recherche concomitante comme une tâche permanente et non seulement comme une évaluation de la phase d'introduction de SwissDRG. La politique de santé ne pourra être durable que si les données pertinentes sont mises à dis- 
position pour fournir les bases décisionnelles requises. Ces bases seront créées en inscrivant la recherche concomitante dans le long terme. Seulement après la mise en place d'une recherche concomitante crédible, la politique aura à disposition un instrument lui permettant de pratiquer une politique de santé à même de garantir à long terme la qualité des soins aux patients, à des coûts raisonnables, tout au long de la chaîne de soins. La FMH va soumettre son concept à SwissDRG SA et nous espérons que les recommandations pourront être mises en œuvre.

\section{Récapitulation des recommandations pour la recherche concomitante}

1. Les travaux portant sur la recherche concomitante à l'occasion de la mise en œuvre de SwissDRG doivent être entrepris sans délai. Il s'agit en effet de s'assurer que les données nécessaires soient collectées à temps avant l'introduction des SwissDRG. Il faut donc établir la recherche concomitante d'ici au $1^{\text {er }}$ janvier 2011 au plus tard.

2. La notion de «recherche concomitante» est à prendre dans un sens très large; elle doit comprendre tous les aspects de la recherche en matière de soins (health services research) dans le système de santé suisse qui sont d'une importance particulière lors de la mise en œuvre de SwissDRG.

3. La recherche concomitante et la recherche en matière de soins doivent être comprises comme une tâche permanente et non seulement comme une évaluation de la phase d'introduction.

4. Il s'agit notamment de distinguer les trois aspects suivants:

a. La sécurité des patients et la qualité des soins.

b. La situation des groupes professionnels concernés.

c. Le système de santé dans son ensemble.

5. Les indicateurs donnés en annexe doivent être traités en considérant qu'ils ont une valeur équivalente.

6. SwissDRG SA doit être désignée comme organisme responsable principal.

7. Le déroulement de l'activité effective de recherche doit être confié au Fonds national suisse de la recherche scientifique.
8 Le financement doit être assuré par différentes sources:

a. les frais d'exploitation facturables des hôpitaux fournisseurs de prestations (notamment pour la statistique médicale et administrative des hôpitaux)

b. un supplément par cas DRG

c. les moyens budgétisés par la Confédération pour l'évaluation des effets de la LAMal

d. d'autres fonds liés à la recherche.

\section{Références:}

1 Cet article est basé sur le rapport suivant présentant un complément d'informations détaillées: Bovier P, Burnand P, Guillain H, Paccaud F, Vader JP, Locher H, Meyer B (2009): Concept pour la recherche concomitante à l'occasion de l'introduction de SwissDRG, www.fmh.ch $\rightarrow$ Tarifs $\rightarrow$ SwissDRG $\rightarrow$ Recherche concomitante.

2 Cf. Bovier P, Burnand B, Guillain H, Paccaud F, Vader JP, Locher H, Meyer B (2009): Concept pour la recherche concomitante à l'occasion de l'introduction de SwissDRG, www.fmh.ch $\rightarrow$ Tarifs $\rightarrow$ SwissDRG $\rightarrow$ Recherche concomitante.

3 Pour la réalisation et la responsabilité de la recherche concomitante, la loi sur l'assurance-maladie (LAMal), l'ordonnance sur l'assurance-maladie (OAMal) ainsi que les relevées statistiques de la Confédération constituent d'importantes bases légales, cf. art. 32 et art. 59d al. 1 lit. b et c LAMal, art. 22 a, art. 23, art 58 OAMal et l'annexe de l'ordonnance du 30 juin 1993 concernant l'exécution des relevées statistiques de la Confédération.

4 Cf. Bovier P, Burnand B, Guillain H, Paccaud F et. al. (2009): Concept pour la recherche concomitante à l'occasion de l'introduction de SwissDRG, www.fmh.ch $\rightarrow$ Tarifs $\rightarrow$ SwissDRG $\rightarrow$ Recherche concomitante.

5 Sur la base du règlement SystemX.ch, cf. http://www.snf.ch/SiteCollectionDocuments/ systemsx_reglement_f.pdf

6 Cf. l'ordonnance concernant les relevées statistiques (RS 431.012.1) et les conditions émises dans l'annexe 2 de l'ordonnance sur les prestations de l'assurance des soins (OPAS), tout particulièrement au sujet du devoir de contribuer au registre.

7 Cf. art. 59d al. 1 lit. b et c LAMal.

8 Contribution par cas au sens de l'art. 49 al. 2 LAMal / Art. 59e OAMal.

9 Cf. art. 32 OAMal. 


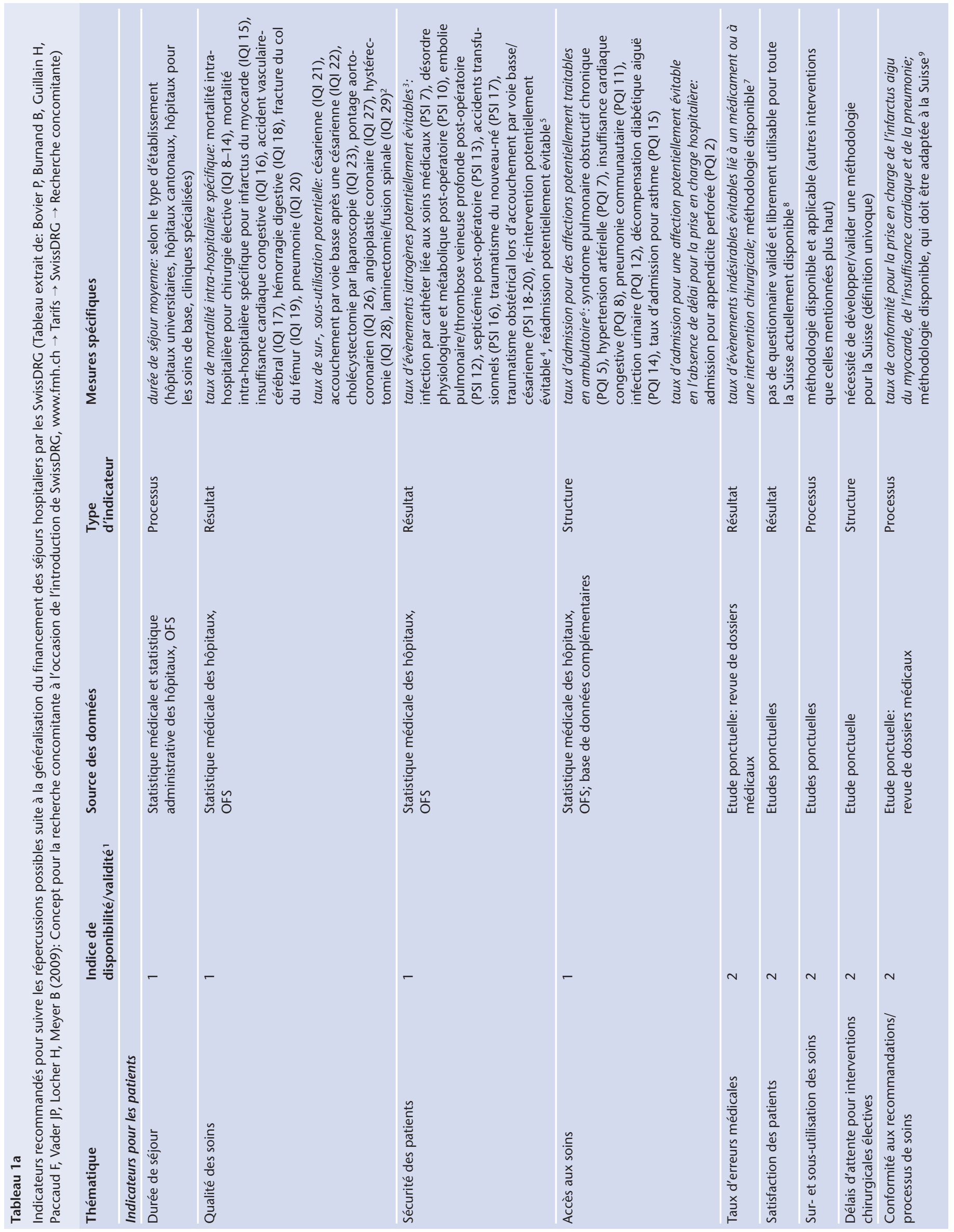




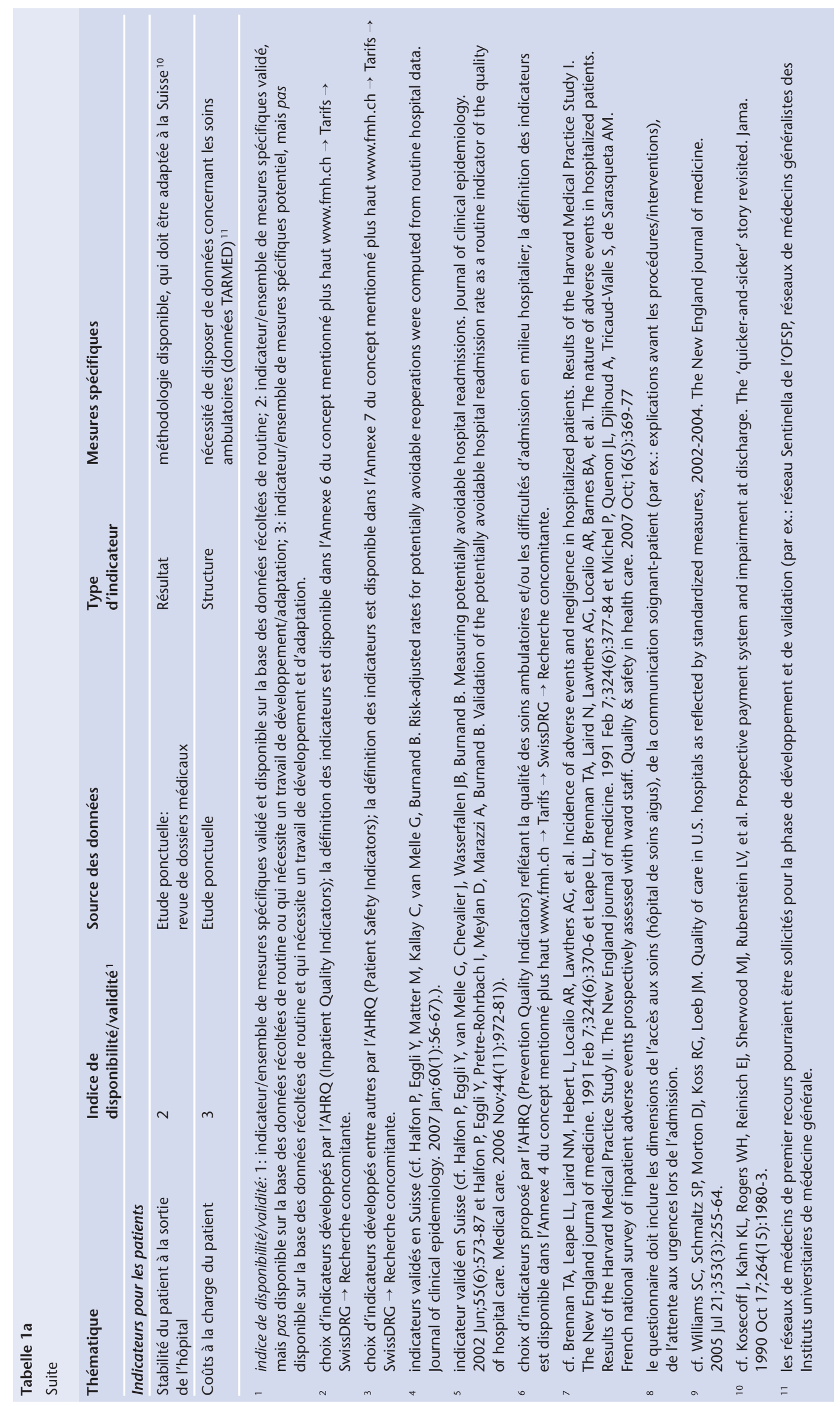




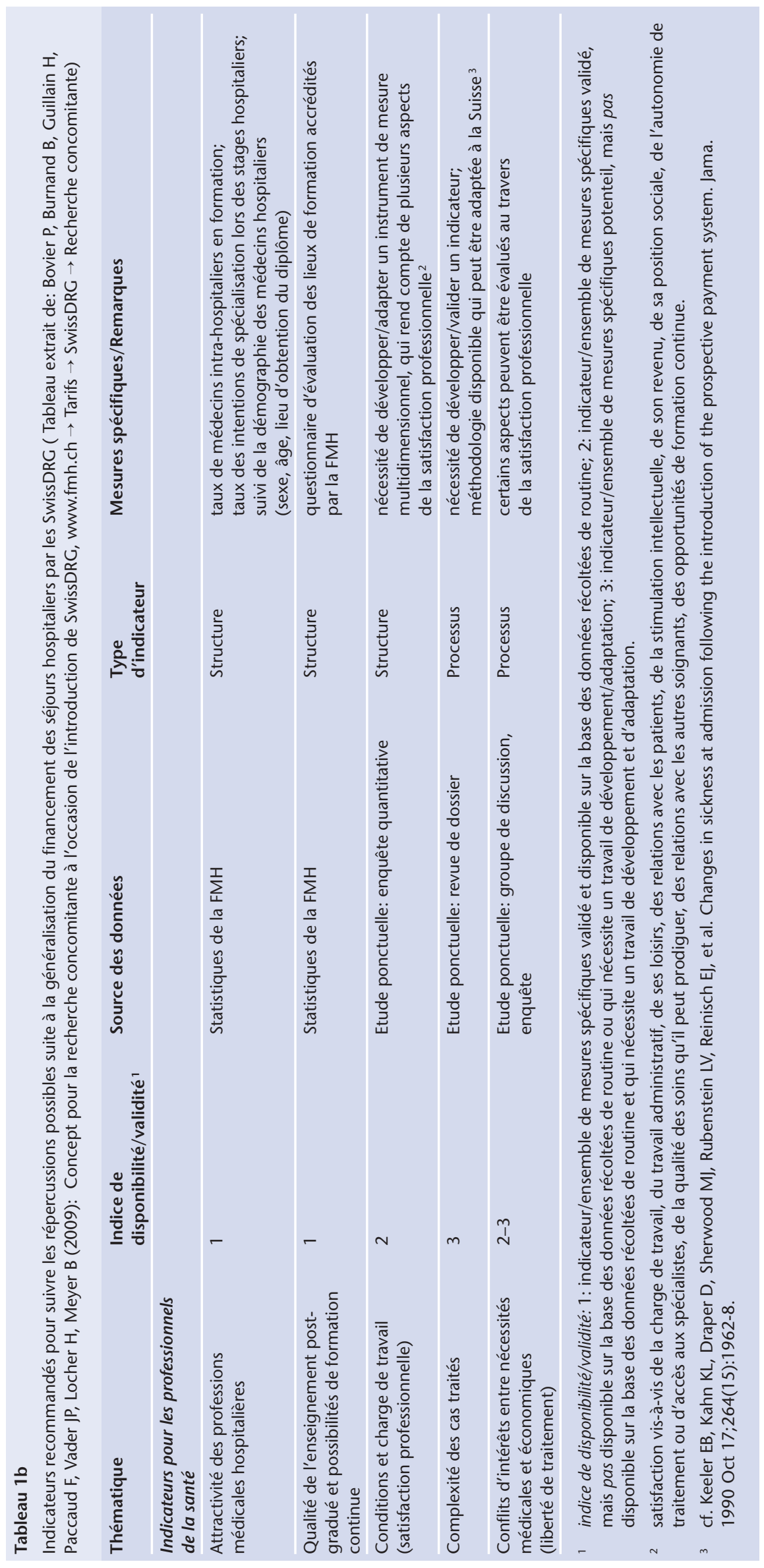




\begin{tabular}{|c|c|c|c|c|c|c|c|c|c|c|c|c|c|c|}
\hline 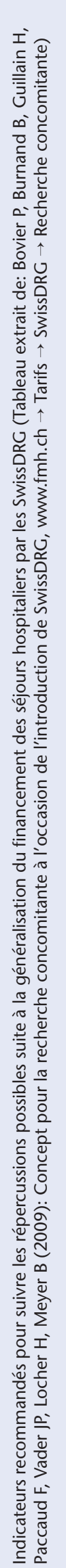 & 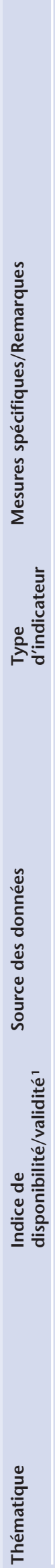 & 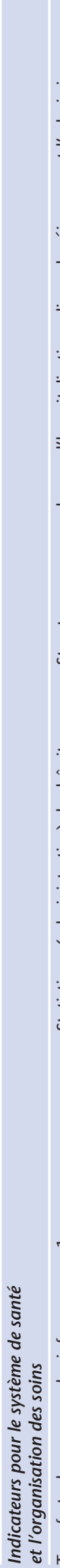 & 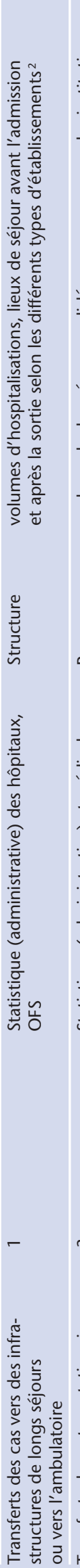 & 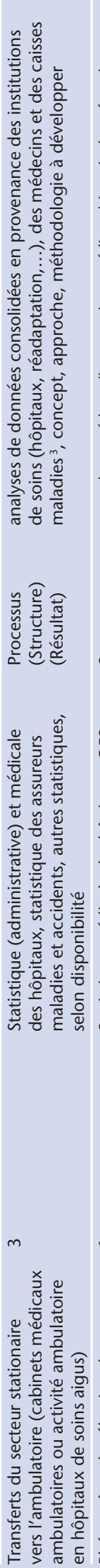 & 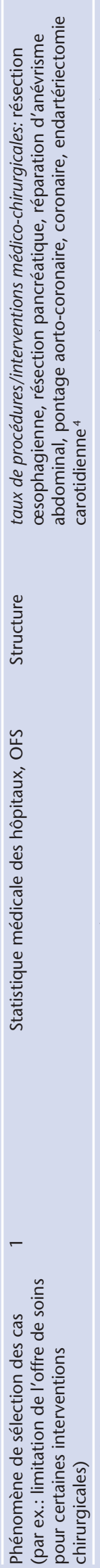 & 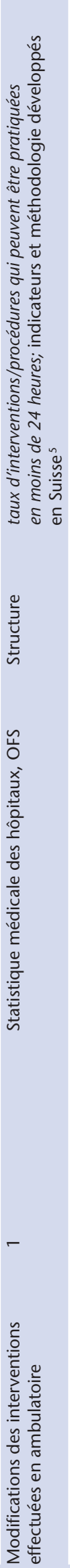 & 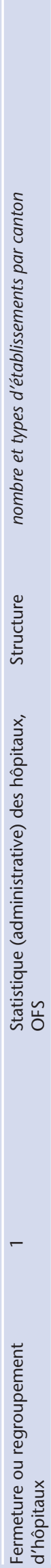 & 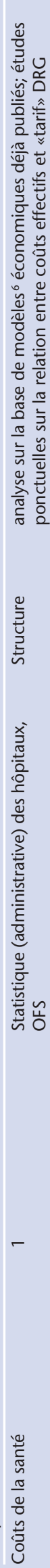 & 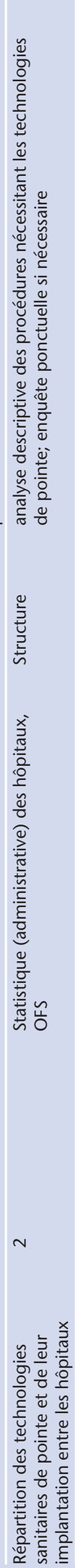 & 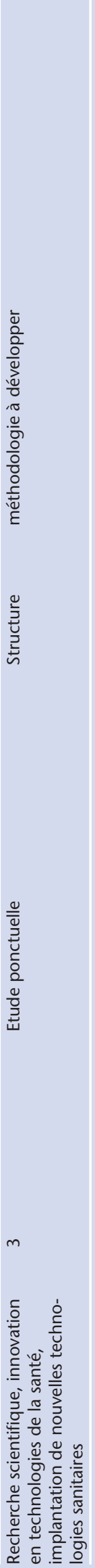 & 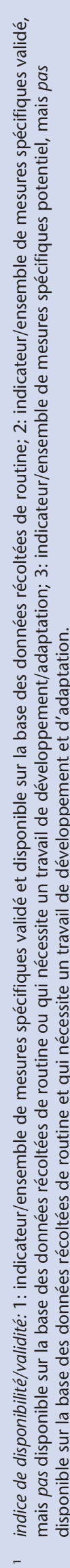 & 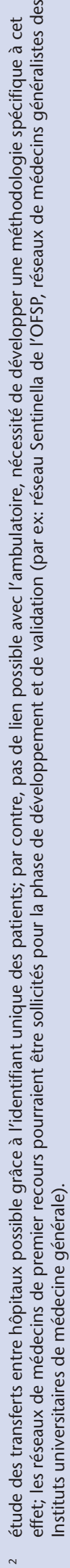 & 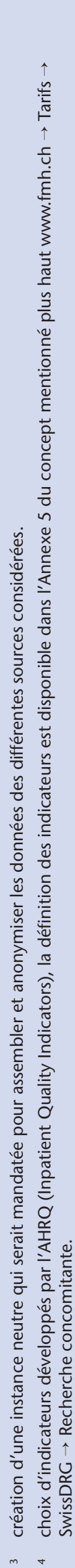 & 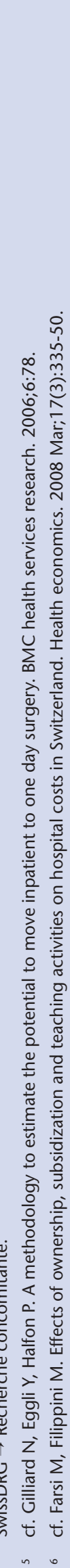 \\
\hline
\end{tabular}

